\title{
Bristol-Myers Squibb acquires potential keys to treating rheumatoid arthritis
}

Bristol-Myers Squibb (BMS) has taken over Padlock Therapeutics in a deal worth up to US\$225 million in upfront and near-term payments, and US $\$ 375$ million in further milestone payments (FIG. 1). In doing so, BMS acquired full rights to Padlock's discovery programme for peptidyl arginine deiminase (PAD) inhibitors, which have shown preclinical promise for autoimmune diseases, particularly rheumatoid arthritis (RA), as well as multiple sclerosis and cancer.

PAD enzymes catalyse the conversion of protein-embedded arginine to citrulline. Citrullination of histones can alter gene expression through epigenetic mechanisms, and non-histone PAD targets include extracellular proteins that are citrullinated during inflammation. These modified proteins are thought to be immunogenic, particularly in RA, in which the presence of anti-citrullinated protein antibodies (ACPAs) is one of the diagnostic criteria. "If you inhibit this path you would be removing the antigen, and that should damp down the inflammation that you see in RA," explains Paul Thompson, one of Padlock's scientific founders, who is now a professor at the University of Massachusetts Medical School, USA. He draws a parallel with coeliac disease - remove gluten, and you remove many of the symptoms.

Thompson's laboratory, then at The University of South Carolina, USA, developed the first potent and specific covalent inhibitors of PAD4 (encoded by PADI4) (Biochemistry 45, 11727-11736; 2006).
These molecules had efficacy in animal models of RA, ulcerative colitis, lupus, atherosclerosis, neonatal hypoxic ischaemia and spinal cord injury.

Although ACPAs are observed only in RA, PAD activity also seems to be critical for the formation of neutrophil extracellular traps, which could explain the efficacy of PAD4 inhibitors in other diseases. These traps are "a unique form of cell death that results in neutrophils basically throwing their DNA out to capture pathogens, creating this meshwork of histones and DNA that can not only capture pathogens but can also act as a landing spot for the formation of blood clots, keep proteases active, help in tissue remodelling, and promote the joint damage that you see in RA," says Thompson. Padlock also in-licensed allosteric PAD4 inhibitors from GlaxoSmithKline; these inhibitors, like Thompson's covalent inhibitors, were effective in preventing the formation of extracellular traps in cell culture (Nat. Chem. Biol. 11, 189-191; 2015) and RA in mouse models (Willis, Ph.D. thesis, University of Colorado, 2012) and are part of the package acquired by BMS.

Citrullination is also important for the non-immunogenic events associated with bone loss in patients with RA. "Osteoclasts are dependent on citrullination and PAD expression for maturation and bone destruction," highlights Anca Catrina, medical head of the early arthritis clinic at the Karolinska University Hospital and Institute, Sweden (Ann. Rheum. Dis. 75, 721-729; 2016). In addition to

\section{Bristol-Myers Squibb acquires Padlock}

Deal type: acquisition

- Acquiring company: Bristol-Myers Squibb

- Acquired company: Padlock Therapeutics
Date: 1 Apr 2016

Value: up to $\$ 600$ million

- US\$225 million in upfront and near-term payments

- US\$375 million in further milestones

Asset characteristics

- Padlock's discovery programme for peptidylarginine deiminase (PAD) inhibitors, which are implicated in rheumatoid arthritis

- PAD inhibitors could also have important roles in coeliac disease, multiple sclerosis and cancer

Figure 1 | Deal snapshot.

destroying bone, she thinks citrullinated protein-containing osteoclasts "may localize the anti-citrullinated protein immunity to the joint," so using PAD inhibitors in patients with early signs of RA could prevent the development of the disease. Thompson agrees, pointing out that ACPAs are often present at least 5 years before the diagnosis of clinical disease, and many ACPA-positive individuals with joint pain will go on to develop RA. Catrina suggests that PAD inhibitors could be particularly important in a new class of patients - those presenting with pain, bone loss and ACPAs (but not overt arthritis) - in which PAD inhibition could prevent the development of RA.

Notably, PADs have also been implicated in other diseases. The expression of various $\mathrm{PAD}$ isoforms is tissue specific. On the basis of studies in mouse models, PAD4 seems to have a role in immune disorders outside the central nervous system, whereas PAD2 is important in multiple sclerosis and cancer. Padlock has primarily been developing inhibitors for PAD4, but PAD2-specific compounds could also be therapeutically relevant. In breast cancer, $\mathrm{PAD} 2$ seems to be involved in the epigenetic regulation of oestrogen receptor-responsive genes, and it is likely that the unusually high levels of citrullinated myelin found in patients with multiple sclerosis are controlled by PAD2. Again, these citrullinated proteins could act as immunoattractants, and PAD inhibitors have shown signs of efficacy in mouse models of multiple sclerosis (J. Med.Chem. 56, 1715-1722; 2013).

Although the therapeutic landscape for RA is already fairly crowded, PAD inhibitors could fill a relatively unoccupied space by preventing disease in patients with early signs of RA. Padlock was planning to initiate first-in-human trials in 2017, so PAD inhibitors could enter clinical trials in the coming year. 